\title{
Erratum to: Occurrence and Environmental Fate of Veterinary Antibiotics in the Terrestrial Environment
}

\author{
Kwon-Rae Kim • Gary Owens • Soon-Ik Kwon • \\ Kyu-Ho So • Deog-Bae Lee $\cdot$ Yong Sik Ok
}

Published online: 20 September 2012

(C) Springer Science+Business Media B.V. 2012

\section{Erratum to: Water Air Soil Pollut (2011) 214:163-174 DOI 10.1007/s11270-010-0412-2}

The original publication of this article (10.1007/s11270010-0412-2) unfortunately contained errors.
In Table 4, the numbers in Martinez-Carballo et al. was in $\mathrm{mg} / \mathrm{kg}$, however, the numbers were incorrectly written in $\mathrm{ug} / \mathrm{kg}$, the numbers from XianGang et al. were incorrect. The corrected table is reproduced here.

The online version of the original article can be found at http://dx.doi.org/10.1007/s11270-010-0412-2.

K.-R. Kim $(\bowtie) \cdot$ S.-I. Kwon • K.-H. So • D.-B. Lee

Climate Change and Agroecology Division,

Department of Agricultural Environment,

National Academy of Agricultural Science, RDA,

150 Suin-ro, Kwonsun-gu,

Suwon 441-707, Republic of Korea

e-mail: Kimkr419@korea.kr

G. Owens

Centre for Environmental Risk Assessment

and Remediation, University of South Australia,

Mawson Lakes,

Adelaide, SA 5095, Australia

Y. S. Ok

Department of Biological Environment,

Kangwon National University,

Chuncheon 200-701, Korea 
Table 4 Variation in the concentration of antibiotic residues $\left(u \mathrm{~g} \mathrm{~kg}^{-1}\right)$ in manure with animal type

The individual compounds in each antibiotics group was given in parenthesis OCT oxitetracycline; $T C$ tetracycline; $S D M$ sulfadimidine; $S M X$ sulfamethoxazole; $S O D$ sulfadimethoxine

\begin{tabular}{lllll}
\hline \multirow{2}{*}{ Manure source } & \multicolumn{2}{l}{ Antibiotic concentration $\left(u \mathrm{~g} \mathrm{~kg}^{-1}\right)$} & \multirow{2}{*}{ Reference } \\
\cline { 2 - 4 } & Tetracyclines & Sulfonamides & Macrolides & \\
\hline Pig & $119(\mathrm{CTC})$ & $9,990(\mathrm{SMZ})$ & 12.4 (TYL) & (Aust et al. 2008) \\
& $100-46,000(\mathrm{CTC})$ & - & - & (Martinez-Carballo et al. 2007) \\
& $210-29,000(\mathrm{OCT})$ & & & \\
& $360-23,000(\mathrm{TC})$ & & - & (Hamscher et al. 2000) \\
Poultry & $880(\mathrm{CTC})$ & - & - & (Bao et al. 2009) \\
& $94.7(\mathrm{CTC})$ & - & 3,700 (TYL) & (Dolliver et al. 2008) \\
& $11,900(\mathrm{CTC})$ & $10,800(\mathrm{SMZ})$ & (Martinez-Carballo et al. 2007) \\
& & $20,000(\mathrm{SDM})$ & & (Hamscher et al. 2000) \\
Cattle & $11(\mathrm{CTC})$ & - & - & (Arikan 2008) \\
\hline
\end{tabular}

